\title{
Inorganic Materials Synthesis Planning with Literature-Trained Neural Networks
}

\author{
Edward Kim, ${ }^{1}$ Zach Jensen, ${ }^{1}$ Alexander van Grootel, ${ }^{1}$ Kevin Huang, ${ }^{1}$ Matthew Staib, ${ }^{2}$ Sheshera Mysore, ${ }^{3}$ \\ Haw-Shiuan Chang, ${ }^{3}$ Emma Strubell, ${ }^{3}$ Andrew McCallum, ${ }^{3}$ Stefanie Jegelka, ${ }^{2}$ and Elsa Olivetti ${ }^{1, *}$ \\ ${ }^{1}$ Dept. of Materials Science and Engineering, Massachusetts Institute of Technology, Cambridge, MA, USA \\ ${ }^{2}$ Dept. of EECS and CSAIL, Massachusetts Institute of Technology, Cambridge, MA, USA \\ ${ }^{3}$ College of Information and Computer Sciences, \\ University of Massachusetts Amherst, Amherst, MA, USA
}

(Dated: February 17, 2019)

\begin{abstract}
Leveraging new data sources is a key step in accelerating the pace of materials design and discovery. To complement the strides in synthesis planning driven by historical, experimental, and computed data, we present an automated method for connecting scientific literature to synthesis insights. Starting from natural language text, we apply word embeddings from language models, which are fed into a named entity recognition model, upon which a conditional variational autoencoder is trained to generate syntheses for arbitrary materials. We show the potential of this technique by predicting precursors for two perovskite materials, using only training data published over a decade prior to their first reported syntheses. We demonstrate that the model learns representations of materials corresponding to synthesis-related properties, and that the model's behavior complements existing thermodynamic knowledge. Finally, we apply the model to perform synthesizability screening for proposed novel perovskite compounds.
\end{abstract}

Recent advances in predicting material properties 1 3], screening synthesizable compounds [4] 6 , and organic reaction prediction [7] have been driven, in part, by the accessibility of machine-readable datasets 10 12 and, consequently, data-driven models. In stark contrast to organic reaction databases [12, the overwhelming majority of inorganic synthesis knowledge lies locked within the text of journal articles [13] and laboratory notebooks [14]. While the latter has been shown as an effective source for guiding successful syntheses, there is currently no general framework for automatically drawing synthesis insights from the literature at large.

Although scientific literature has previously been used to illuminate patterns in nanoscale morphologies [13], device performances [15], and apparatus parameters [16], each of these efforts have required tailored data representations and algorithms. To rapidly translate literature knowledge into synthesis planning resources without finetuned adjustments, transfer learning across different materials systems is necessary [13]. Thus, leveraging data from broad volumes of scientific literature is a critical step towards capturing synthesis trends and extending them to unknown materials.

In this work, we present an automated method for connecting scientific literature to insights for materials synthesis planning. We show that an unsupervised conditional variational autoencoder (CVAE) [17-19] can generate synthesis predictions for a variety of materials, including materials unknown to the model. This CVAE learns directly from the materials synthesis literature and produces an internal representation of precursors which corresponds to physical and chemical trends without receiving any explicit domain knowledge. We use the liter-

\footnotetext{
* Corresponding author: elsao@mit.edu
}

ature knowledge captured by the CVAE to complement first-principles techniques in materials screening tasks.

To accelerate the efforts of the materials science community, we open-source several key resources used in this work [20]: We release context-sensitive embeddings from language models (ELMo) that have been adapted for materials science text [21] along with a pre-trained FastText word embedding model for materials science [22]. Each of these embedding models has been trained on a collection of over 2.5 million materials science journal articles [13, 23. Finally, we provide over two hundred annotated literature synthesis routes for named entity recognition (NER) tasks, such as identifying reaction conditions and materials.

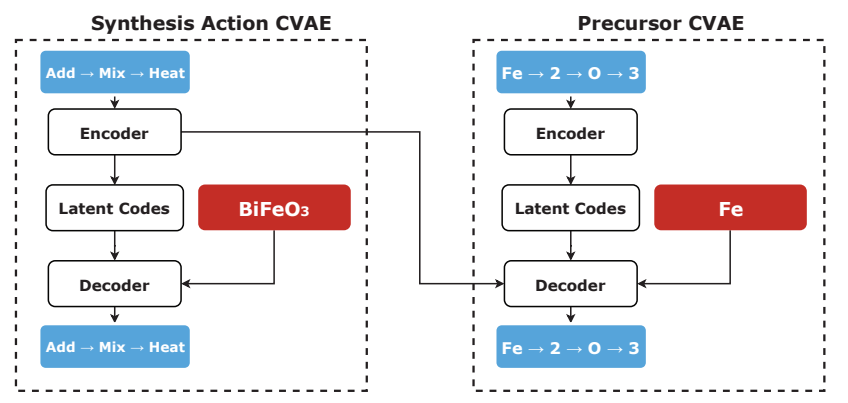

FIG. 1. Schematic diagram of the CVAE architecture. The model consists of two joined CVAEs used for learning synthesis actions and precursors, respectively. The Synthesis Action CVAE learns distributions of synthesis actions sequences conditioned on target materials. The Precursor CVAE learns distributions of precursor formulas conditioned on both a target element and an encoded representation of the jointly-observed synthesis action sequence. Target materials are represented by FastText embeddings, and all other inputs to the model are sequences of one-hot vectors. 
We first describe our automated workflow. After a recurrent neural network 24 identifies synthesis sections of journal articles, context-sensitive ELMo word embeddings are computed and passed into another recurrent neural network which performs NER to identify precursors, synthesis target materials, and synthesis actions. Then, a CVAE model, shown in Figure 1, is trained to learn representations of synthesis routes from the named entities in an unsupervised manner. More details on these methods are provided in the Supplementary Methods.

To maximize the opportunity for transfer learning of synthesis trends, we choose a broad definition for synthesis routes that requires minimal assumptions. For a given target material $m$, a synthesis route $S_{m}^{i}$ is a 2-tuple consisting of a sequence of $n$ synthesis actions $\left(a_{1}, a_{2}, \ldots, a_{n}\right)$ acting on a set of $l$ precursors $\left\{p_{1}, \ldots, p_{l}\right\}$,

$$
S_{m}^{i}=\left(\left(a_{k}\right)^{n},\left\{p_{j}\right\}^{l}\right)
$$

and in general, a single target material $m$ may have $N>$ 1 valid synthesis routes and thus $S_{m}^{i}$ represents the $i$ th valid synthesis route for $m$. We also define precursors $p$ as "element sources," such that they are materials sharing an element with $m$. The CVAE model is then constructed to model the following distributions,

$$
\begin{aligned}
& \mathbb{P}\left(\left(a_{k}\right)^{n} \mid \theta_{a}, m\right) \\
& \mathbb{P}\left(p_{j} \mid \theta_{p}, e_{j},\left(a_{k}\right)^{n}\right)
\end{aligned}
$$

where $\theta_{a}$ and $\theta_{p}$ are model parameters for the synthesis action and precursor CVAEs, respectively, and $e_{j}$ is the shared element between a precursor $p_{j}$ and target material $m$ (e.g., titanium).

Since CVAEs are generative models, novel synthesis actions and precursors can be generated by sampling from a Gaussian prior distribution [17]. Critically, we represent $m$ by FastText word embeddings which enable the transfer of synthesis trends between existing and novel materials by leveraging literature-based similarity.

TABLE I. Generated precursors for $\mathrm{InWO}_{3}$ and $\mathrm{PbMoO}_{3}$, drawn from the CVAE model. The CVAE model was trained on synthesis routes published during or before 2005. A more detailed version is available in the Supplemental Results.

Target Material

Precursors

\begin{tabular}{lr}
\hline \hline & $\mathrm{In}_{2} \mathrm{~S}_{3}+\mathrm{WCl}_{4}$ \\
$\mathrm{InWO}_{3}$ & $\mathrm{In}\left(\mathrm{NO}_{3}\right)_{3}+\mathrm{WCl}_{4}$ \\
& $\mathrm{In}_{2} \mathrm{O}_{3}+\mathrm{WO}_{2}$ \\
& $\mathrm{In}_{2} \mathrm{O}_{3}+\mathrm{WN}$ \\
& ${ }^{\dagger} \mathrm{InCl}_{3}+\mathrm{Na}_{2} \mathrm{WO}_{4}$ \\
\hline $\mathrm{PbMoO}_{3}$ & $\mathrm{PbCl}_{2}+\mathrm{MoCl}_{2}$ \\
& $\mathrm{PbSO}_{4}+\mathrm{MoCl}_{2}$ \\
& ${ }^{\ddagger} \mathrm{PbO}+\mathrm{MoO}_{2}$ \\
\hline \hline
\end{tabular}

† Precursors match Kamalakkannan et al. (2016) [25].

$\ddagger$ Precursors match Takatsu et al. (2017) [26].

To demonstrate the applicability of our CVAE method, we construct a dataset of approximately 51,000 synthe- sis action sequences and 116,000 precursors via a general set of search terms ("perovskite + thermoelectric + multiferroic + photovoltaic + solar + nano + cathode") and apply our neural network pipeline. We investigate the effectiveness of the CVAE model in synthesis planning by performing a publication-year-split experiment, where the model is trained only on syntheses published prior to 2005 ( $\sim 2800$ syntheses). We apply the model in predicting precursors for materials that were unseen during training, are computationally predicted as stable perovskites [1], and only recently appear in the literature: $\mathrm{InWO}_{3}$ and $\mathrm{PbMoO}_{3}$, first reported in 2016 and 2017, respectively [25, 26]. Table 1 shows a report of the data generated by sampling from the CVAE's Gaussian prior distributions, where the CVAE suggests the precursors for both materials (see Table ?? for additional details). The CVAE model is thus capable of predicting synthesis precursors while relying only on literature knowledge from more than a decade prior to the literature-reported syntheses of these materials. Trial-and-error (or random) precursor selection is substantially less efficient, as the number of possible precursor sets for each material is in the hundreds. Thus literature-driven models may greatly accelerate future synthesis attempts of novel materials.

During the data generation process, the CVAE model proposes several plausible syntheses beyond the literature-matching samples. To the best of the authors' knowledge, the only reported synthesis of $\mathrm{InWO}_{3}$ is via a solution-phase route. However, the CVAE model suggests that solid state synthesis of $\mathrm{InWO}_{3}$ may be possible, using $\mathrm{In}_{2} \mathrm{O}_{3}$ and either $\mathrm{WO}_{2}$ or $\mathrm{WN}$ as precursors. Such syntheses may be feasible, as they are thermodynamically favorable (using data at $0 \mathrm{~K}$ and $0 \mathrm{~atm}$ from OQMD) [10]:

$$
\begin{aligned}
\mathrm{In}_{2} \mathrm{O}_{3}+2 \mathrm{WO}_{2} & \longrightarrow 2 \mathrm{InWO}_{3}+2 \mathrm{O}_{2} \\
\Delta \mathrm{H} & =-158 \mathrm{~kJ} / \mathrm{mol} \\
\mathrm{In}_{2} \mathrm{O}_{3}+2 \mathrm{WN} & \longrightarrow 2 \mathrm{InWO}_{3}+\mathrm{N}_{2} \\
\Delta \mathrm{H} & =-930 \mathrm{~kJ} / \mathrm{mol}
\end{aligned}
$$

We do note, however, that these simple thermodynamic analyses can only be used as rough guidelines. Besides the limitations of estimating an overall thermochemical reaction for the synthesis, along with extrapolation from STP conditions, kinetic effects are not considered here. Indeed, while it is common to mix binary oxide precursors in solid state syntheses of ternary (or quaternary, etc.) oxides, the use of nitride precursors is far less common due to the high bond energies of many nitride compounds [27]. To achieve a clearer understanding of kinetic effects, experimental verification would be required alongside a model which incorporates reaction conditions (e.g., temperatures), and this is an area for future work.

In the suggested recipes for $\mathrm{PbMoO}_{3}$, the CVAE model suggests a solution-phase route using $\mathrm{PbSO}_{4}$ and $\mathrm{MoCl}_{2}$, both of which are soluble under acidic conditions. This may provide another viable path towards synthesizing 
$\mathrm{PbMoO}_{3}$, which has only been realized so far by solid state synthesis methods [26]. Thus, the CVAE may be used as a source of suggestions for synthesis planning, although we stress that these suggestions still need human evaluation, further analysis, and cannot be applied "out of the box."

Despite the fact that chemical knowledge is never given to the CVAE model, solubility rules emerge from the model results. To demonstrate this, we generate $\mathrm{W}$ bearing precursors for $\mathrm{InWO}_{3}$ conditioned on two representative action sequences sampled from the Synthesis Action CVAE: A solid-state synthesis (mix, grind, calcine, press, sinter, cool) and a solution-phase synthesis (add, dissolve, stir, heat, wash, dry). Following this, we generate 10,000 CVAE-suggested precursors. The most common W-bearing precursor generated for the solidstate synthesis is the water-insoluble $\mathrm{WO}_{3}$, while the the most common precursor generated for the solution-phase synthesis is the highly-soluble $\mathrm{Na}_{2} \mathrm{WO}_{4}$. The differences of these precursor likelihoods in each case is substantial, with $-16 \%$ and $+21 \%$ changes to the likelihoods of the CVAE suggesting $\mathrm{WO}_{3}$ and $\mathrm{Na}_{2} \mathrm{WO}_{4}$, respectively, when switching from conditioning on solid-state to solutionphase synthesis actions.

This effect of learning precursor trends from the literature is further demonstrated upon inspecting latent codes learned by the model. Since the CVAE learns conditional distributions, the input precursors are projected into a degenerate latent space, where the degeneracy is split by the conditional input received by the decoder. By investigating several examples (see Figure ??), we find that the CVAE learns to group precursors with similar synthesis-relevant properties, including insoluble binary oxides, water-soluble polyanion compounds, and pure/alloyed metals. This suggests that the CVAE model is capable of capturing chemical intuition and composition-driven similarity solely by joint observations of precursors, synthesis actions, and target materials. Despite the lack of "negative" data in the literature, the diversity of published synthesis literature is sufficient to drive the CVAE model in learning meaningful representations of precursors.

To emphasize the particular nature of synthesis planning via a literature-trained model, we contrast suggested precursors by the CVAE model with thermodynamic stability computations from OQMD [10]. Figure 2 a shows a graph representation of the chemical space spanned by all CVAE-suggested precursors, where graph vertices are precursors and graph edges represent thermodynamic two-phase equilibria [28]. Clearly, the CVAE is not simply suggesting all thermodynamically-viable precursors, as there is a substantial set of precursors never suggested by the CVAE (blue vertices versus red vertices). Indeed, the CVAE has filtered precursors from a set of 73 possible precursors to only 27 . Additionally, Figures $2 \mathrm{~b}$ and $2 \mathrm{c}$ show that the CVAE is not selecting precursors in correspondence with isolated thermodynamic metrics: the CVAE's suggestions are explained neither by thermody- namic reactivity (i.e., the number of relevant two-phase equilibria with respect to other precursors) nor individual precursor stability (i.e., formation energy). This suggests that there is a meaningful difference between the thermodynamically-driven and literature-driven synthesis planning methods. While the former probes the realm of physical possibility, the latter emphasizes practical choices and historical trends.

We next train the CVAE model on our full dataset, using no publication-year cutoffs. To investigate the capability of the model for suggesting syntheses of a novel, never-before-synthesized material, we consider novel $\mathrm{ABO}_{3}$ perovskite materials proposed by Balachandran et al. 29. These proposed perovskites have not previously been synthesized, and have high thermodynamic stability as measured by energy differences against their convex hulls. We note that $\mathrm{ABO}_{3}$ perovskites are used here as a representative example due to their chemical variety and diverse range of properties, but the CVAE model does indeed generalize to other categories of materials (see Table ??).

$\mathrm{HgZrO}_{3}$ is one such example of a thermodynamically stable, unsynthesized perovskite material [29], and we perform synthesis predictions using the CVAE model (see Table ??). We find that the CVAE proposes solid-state syntheses which appear to be thermodynamically reasonable:

$$
\begin{aligned}
\mathrm{HgO}+\mathrm{ZrC}+2 \mathrm{O}_{2} & \longrightarrow \mathrm{HgZrO}_{3}+\mathrm{CO}_{2} \\
\Delta \mathrm{H} & =-1340 \mathrm{~kJ} / \mathrm{mol} \\
\mathrm{HgO}+\mathrm{ZrO}_{2} & \longrightarrow \mathrm{HgZrO}_{3} \\
\Delta \mathrm{H} & =-0.29 \mathrm{~kJ} / \mathrm{mol}
\end{aligned}
$$

Again, we emphasize that thermodynamic analyses are often insufficient to evaluate reaction plausibility. As an additional utility for evaluating generated synthesis parameters, we develop a similarity metric based on the latent codes learned by the CVAE. By measuring nearestneighbors of latent codes for the recipe using mercuric oxide and zirconium carbide, we find that the two closest literature recipes are for solid-state syntheses of $\mathrm{SrZrO}_{3}$ and $\mathrm{BaAl}_{2} \mathrm{O}_{4}$ (see Figure ??). Besides providing insight into which observed literature examples "inspired" this particular prediction, we are also led to further insights on precursor selections. $\mathrm{ZrC}$ is an uncommon choice of precursor, but carbonate precursors are readily used in solid-state syntheses. Indeed, both of the near-neighbor syntheses for $\mathrm{HgZrO}_{3}$ use carbonate precursors rather than carbides.

While similarity methods have previously been produced for materials (e.g., based on crystal structures) [30], the CVAE incorporates synthesis knowledge to produce a distinct measure of similarity. Indeed, from a structural point of view, it would not be expected that $\mathrm{SrZrO}_{3}$ and $\mathrm{BaAl}_{2} \mathrm{O}_{4}$ should have high similarity to $\mathrm{HgZrO}_{3}$, since all three materials form ground- 

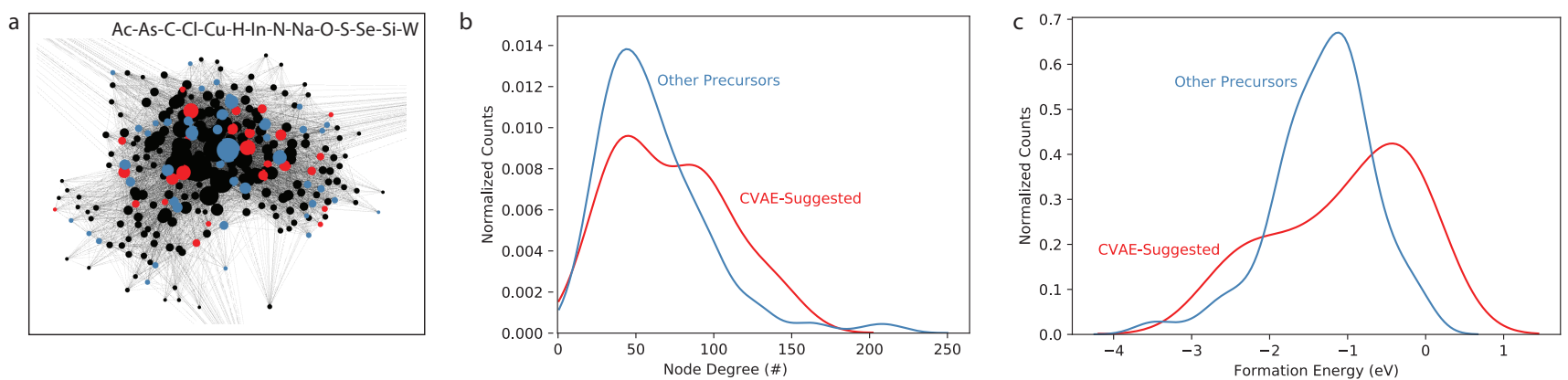

FIG. 2. Graph representation of a phase diagram for $\mathrm{InWO}_{3}$ precursor chemical space [28], using data from 1000 precursor sets generated by the CVAE. a) Zoomed-in view of phase diagram graph for the 14-element chemical system. Red ( $\mathrm{n}=27)$ and blue $(\mathrm{n}=46)$ colored nodes are precursors for $\mathrm{InWO}_{3}$ (i.e., materials containing $\mathrm{In}$ or $\mathrm{W}$ ), and all other material nodes are black $(\mathrm{n}=198)$. Red nodes are suggested at least once by the CVAE, and blue nodes are never suggested by the CVAE. Graph edges represent two-phase equilibria, as determined by OQMD [10, 28, and node sizes are proportional to node degree. b) Distributions of graph node degrees (i.e., number of two-phase equilibria) for CVAE-suggested and non-suggested precursors. c) Distributions of formation energies for CVAE-suggested and non-suggested precursors.

state structures in different crystal systems (orthorhombic, hexagonal, and cubic, respectively).

Moreover, rather than measuring similarity against entire materials, the CVAE-based metric operates at the level of individual reported (or generated) synthesis routes. Since nearest-neighbor search is computationally inefficient in high dimensional spaces, the dimensionality reduction imposed by the CVAE enables this "latent citation" model to be used as a rapid, data-driven synthesis planning method.

Finally, we present results for synthesis screening using the CVAE model to suggest syntheses for numerous $\mathrm{ABO}_{3}$ suggested by Balachandran et al. 29. The CVAE was used to generate syntheses with ten data generation attempts per compound, and only compounds which had at least one suggested synthesis route with commerciallyavailable precursors were considered to have passed the test, which is the same criterion used by Segler et al. 7] to evaluate retrosynthetic routes for organic molecules.

Figure 3 shows a grid of possible A-site and B-site atoms for $\mathrm{ABO}_{3}$ perovskite materials, with screened compounds represented by highlighted combinations of Asite and B-site atoms. While a joint machine learning and density functional theory method [29] selects a set of materials which are thermodynamically stable in the perovskite form, the further-imposed synthesis screening selects a subset that is most readily synthesizable based on existing literature knowledge. From a set of 83 proposed $\mathrm{ABO}_{3}$ perovskite compounds, the CVAE has selected a subset of only 19.

The CVAE model, combined with the rest of our neural network workflow, enables a new axis of synthesis screening which complements existing domain knowledge [5, 29. By incorporating and extending patterns in historical literature, materials which are theoretically synthesizable can be rapidly and automatically filtered by their practical synthesizability. While the methods pre-

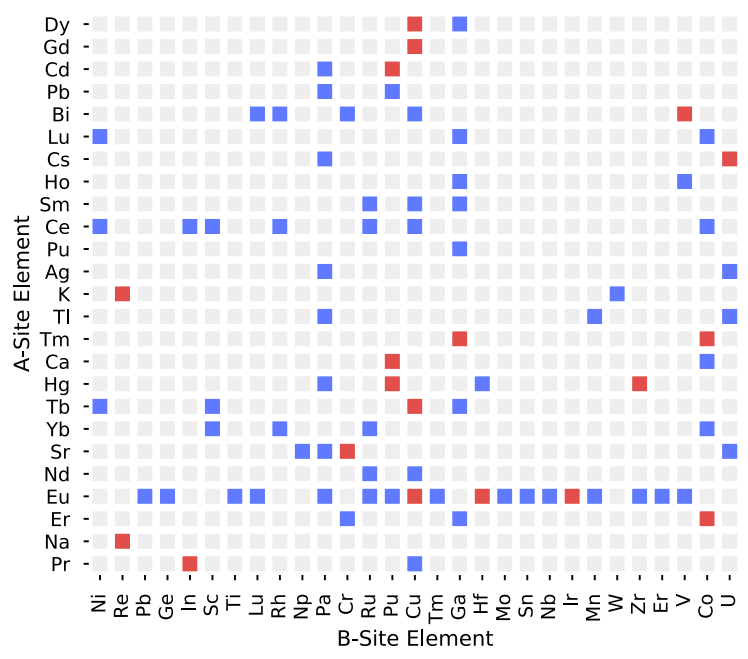

FIG. 3. Unsynthesized $\mathrm{ABO}_{3}$ perovskite compounds, labelled by their A-site and B-site elements. Colored-in squares are perovskites predicted to be stable [29. Red and blue colors correspond to compounds which passed or failed the CVAE screening, respectively.

sented in this paper are applicable to various materials systems and synthesis methods, we recognize that our broad representation of synthesis routes omits information such as temperatures, solvents, and morphologies, and additionally assumes that there is a one-to-one relation between elements in precursors and targets. We thus believe that promising future work lies in the direction of generative models with narrower scope, but finer-grained detail. For example, limiting the dataset to solvothermal syntheses may facilitate prediction of solvent choices, solvothermal reaction temperatures, and 
dwell times. This additional domain knowledge may be incorporated by filtering proposed synthesis parameters [7] or constraining model outputs [31]. Motivated by these possibilities, our open-source NER annotations include the necessary labels (e.g., reaction conditions) to enable these future studies.

We would like to acknowledge funding from the National Science Foundation Award 1534340, DMREF that provided support to make this work possible, support from the Office of Naval Research (ONR) under Contract No. N00014-16-1-2432, the MIT Energy Initiative, and NSF CAREER \#1553284. Early work was collaborative under the Dept. of Energy's Basic Energy Science Program through the Materials Project under Grant No. EDCBEE. This work was also partly funded by the MIT-Sensetime Alliance on Artificial Intelligence. We would also like to acknowledge valuable feedback from Rafael Jaramillo, Gerbrand Ceder, Olga Kononova, Wenhao Sun, Haoyan Huo, and Tanjin He.
[1] T. Xie and J. C. Grossman, Physical Review Letters 120, 145301 (2018).

[2] T. D. Huan, A. Mannodi-Kanakkithodi, and R. Ramprasad, Physical Review B 92, 014106 (2015).

[3] O. Isayev, D. Fourches, E. N. Muratov, C. Oses, K. Rasch, A. Tropsha, and S. Curtarolo, Chemistry of Materials 27, 735 (2015).

[4] B. Meredig, A. Agrawal, S. Kirklin, J. E. Saal, J. Doak, A. Thompson, K. Zhang, A. Choudhary, and C. Wolverton, Physical Review B 89, 094104 (2014).

[5] M. Aykol, V. I. Hegde, S. Suram, L. Hung, P. Herring, C. Wolverton, and J. S. Hummelshøj, arXiv preprint arXiv:1806.05772 (2018).

[6] K. Kim, L. Ward, J. He, A. Krishna, A. Agrawal, and C. Wolverton, Physical Review Materials 2, 123801 (2018).

[7] M. H. Segler, M. Preuss, and M. P. Waller, Nature 555, 604 (2018).

[8] H. Gao, T. J. Struble, C. W. Coley, Y. Wang, W. H. Green, and K. F. Jensen, ACS Central Science (2018).

[9] C. W. Coley, R. Barzilay, T. S. Jaakkola, W. H. Green, and K. F. Jensen, ACS Central Science 3, 434 (2017).

[10] J. E. Saal, S. Kirklin, M. Aykol, B. Meredig, and C. Wolverton, Jom 65, 1501 (2013).

[11] A. Jain, S. P. Ong, G. Hautier, W. Chen, W. D. Richards, S. Dacek, S. Cholia, D. Gunter, D. Skinner, G. Ceder, et al., APL Materials 1, 011002 (2013).

[12] J. Goodman, "Computer software review: Reaxys," (2009).

[13] E. Kim, K. Huang, A. Saunders, A. McCallum, G. Ceder, and E. Olivetti, Chemistry of Materials 29, 9436 (2017).

[14] P. Raccuglia, K. C. Elbert, P. D. Adler, C. Falk, M. B. Wenny, A. Mollo, M. Zeller, S. A. Friedler, J. Schrier, and A. J. Norquist, Nature 533, 73 (2016).

[15] L. Ghadbeigi, J. K. Harada, B. R. Lettiere, and T. D. Sparks, Energy \& Environmental Science 8, 1640 (2015).

[16] S. R. Young, A. Maksov, M. Ziatdinov, Y. Cao, M. Burch, J. Balachandran, L. Li, S. Somnath, R. M. Patton, S. V. Kalinin, et al., Journal of Applied Physics 123, 115303 (2018).

[17] D. P. Kingma and M. Welling, in International Conference on Learning Representations (2014).

[18] K. Sohn, H. Lee, and X. Yan, in Advances in Neural Information Processing Systems (2015) pp. 3483-3491.

[19] R. Gómez-Bombarelli, J. N. Wei, D. Duvenaud, J. M. Hernández-Lobato, B. Sánchez-Lengeling, D. Sheberla, J. Aguilera-Iparraguirre, T. D. Hirzel, R. P. Adams, and A. Aspuru-Guzik, ACS Central Science 4, 268 (2018).

[20] www.github.com/olivettigroup/ materials-synthesis-generative-models

[21] M. E. Peters, M. Neumann, M. Iyyer, M. Gardner, C. Clark, K. Lee, and L. Zettlemoyer, in Proceedings of the 2018 Conference of the North American Chapter of the Association for Computational Linguistics: Human Language Technologies, Volume 1 (Long Papers) (2018).

[22] P. Bojanowski, E. Grave, A. Joulin, and T. Mikolov, Transactions of the Association for Computational Linguistics 5, 135 (2017).

[23] E. Kim, K. Huang, A. Tomala, S. Matthews, E. Strubell, A. Saunders, A. McCallum, and E. Olivetti, Scientific Data 4, 170127 (2017).

[24] J. Chung, C. Gulcehre, K. Cho, and Y. Bengio, in NIPS 2014 Workshop on Deep Learning, December 2014 (2014).

[25] J. Kamalakkannan, V. Chandraboss, and S. Senthilvelan, World Scientific News 58, 97 (2016).

[26] H. Takatsu, O. Hernandez, W. Yoshimune, C. Prestipino, T. Yamamoto, C. Tassel, Y. Kobayashi, D. Batuk, Y. Shibata, A. M. Abakumov, et al., Physical Review B 95, 155105 (2017).

[27] W. Sun, A. Holder, B. Orvañanos, E. Arca, A. Zakutayev, S. Lany, and G. Ceder, Chemistry of Materials 29, 6936 (2017).

[28] V. I. Hegde, M. Aykol, S. Kirklin, and C. Wolverton, arXiv preprint arXiv:1808.10869 (2018).

[29] P. V. Balachandran, A. A. Emery, J. E. Gubernatis, T. Lookman, C. Wolverton, and A. Zunger, Physical Review Materials 2, 043802 (2018).

[30] L. Yang and G. Ceder, Physical Review B 88, 224107 (2013).

[31] M. J. Kusner, B. Paige, and J. M. Hernández-Lobato, in International Conference on Machine Learning (2017) pp. 1945-1954.

[32] F. Chollet et al., "Keras," (2015).

[33] M. Abadi, P. Barham, J. Chen, Z. Chen, A. Davis, J. Dean, M. Devin, S. Ghemawat, G. Irving, M. Isard, et al., in $O S D I$, Vol. 16 (2016) pp. 265-283.

[34] S. P. Ong, W. D. Richards, A. Jain, G. Hautier, M. Kocher, S. Cholia, D. Gunter, V. L. Chevrier, K. A. Persson, and G. Ceder, Computational Materials Science 68, 314 (2013).

[35] M. C. Swain and J. M. Cole, Journal of chemical information and modeling 56, 1894 (2016).

[36] S. Katyayan and S. Agrawal, Journal of Materials Science: Materials in Electronics 28, 18442 (2017).

[37] A.-K. Larsson, R. Withers, J. Perez-Mato, J. F. Gerald, P. J. Saines, B. J. Kennedy, and Y. Liu, Journal of Solid State Chemistry 181, 1816 (2008). 